\title{
Conservadorismo e rigidez na política monetária: uma estimativa da função de reação do BCB (2000-2007)
}

\section{ANDRÉ DE MELO MODENESI*}

Conservatism and rigidity in monetary policy.

This paper aims at evaluating the conduction of monetary policy after the adoption of inflation targeting. Formation of Selic rate is modeled by estimating a reaction function of the BCB. Results show an excessive degree of interest rate smoothing and a high level of equilibrium interest rate. This evidence supports the belief that Selic rate's formation is ruled by a conservative behavior. The conservative conduction of monetary policy is related to two distinct features of BCB's reaction function: i) the great weight of autoregressive components; and, chiefly, ii) a very high level of the equilibrium interest rate. The main conclusion is that, all remaining unchanged, the interest rate would hardly be reduced in a satisfactory way. Massive and chronic deflation would be needed if Selic were to reach a reasonable level, closer to that of rates in the rest of the world. This evidences the need for a debate on the adequacy of current stabilization strategy.

Keywords: Taylor rule; Selic; conservatism.

JEL Classification: E43; E58.

\section{INTRODUÇÃO}

Quinze anos após a conquista da estabilidade de preços, ainda não se verificou uma queda suficiente das taxas de juros reais no país. De início, a manutenção da

\footnotetext{
* Professor do Instituto de Economia da Universidade Federal do Rio de Janeiro (IE/UFRJ) e Pesquisador do CNPq. E-mail: amodenesi@gmail.com. Versões anteriores desse artigo foram apresentadas no I Encontro Internacional da Associação Keynesiana Brasileira (Unicamp, 4/2008), na $10^{\text {th }}$ International Post Keynesian Conference (Kansas City, 6/2008) e no XXVI Encontro Nacional de Economia da ANPEC (Salvador, 12/2008). O autor agradece a colaboração de Rui L. Modenesi, Fabio S. Erber, Luiz F. R. de Paula, e os comentários de Manoel C. Pires, Salvador W. Vianna, Marco A. Cavalcanti, Roberta O. Guimarães e Felix Manhiça. Submetido: Janeiro 2009; Aprovado: Janeiro 2010.
} 
taxa básica de juros (Selic) em níveis elevados era justificada pelo Banco Central do Brasil (BCB) como meio de se compensar o déficit em conta-corrente (quase US\$ 35 bilhões, em 1998) ou de se preservarem as reservas internacionais. A política fiscal expansionista do primeiro governo FHC também era apontada como uma das causas da rigidez da política monetária.

O abandono da âncora cambial, em 1999, a melhoria nas contas externas e a mudança na instância da política fiscal - com superávit primário de cerca de $4 \%$ do PIB - não foram suficientes para reduzir substancialmente a Selic. De fato, a Selic real caiu de forma não desprezível no período de 2000 a 2007, tendo sua média se aproximado de $10 \%$ a.a. No entanto, ainda se trata de um patamar muito alto. A taxa básica de juros real média praticada pelos países emergentes, por exemplo, foi de cerca de $2.7 \%$ a.a., no mesmo período. Isto é, a taxa de juros no Brasil é quase quatro vezes maior do que a dos países emergentes. Consequentemente, é bastante difundida a visão de que o BCB é muito conservador na fixação da Selic.

Este artigo tem como objetivo avaliar a condução da política monetária no Brasil, após a flexibilização cambial e a adoção do regime de metas de inflação (RMI). Na próxima seção é feita uma resenha da literatura sobre a chamada regra de Taylor. Na terceira seção, o processo de determinação da taxa de juros é sistematizado por meio da estimação de uma regra de reação do BCB, entre a adoção do RMI e o ano de 2007.

$\mathrm{Na}$ quarta seção destaca-se que a evidência empírica corrobora a ideia de que a formação da taxa de juros no país vem-se fundamentando em uma postura excessivamente conservadora por parte do BCB. A principal conclusão é que, mantido o prevalecente conservadorismo na condução da política monetária, a taxa de juros dificilmente seria reduzida de forma satisfatória pelo BCB. As simulações realizadas indicam que seria necessária uma deflação crônica e de grande magnitude para que a Selic caísse significativamente.

A constatação desse fato é o primeiro passo para o reconhecimento de que, no caso brasileiro, o RMI tem-se mostrado pouco adequado. Nas considerações finais destaca-se que a política anti-inflacionária tem resultado em elevadíssima taxa de sacrifício - medida não apenas pelo alto desemprego e o relativo desaquecimento da economia, mas, também, pelo enorme custo fiscal da política monetária. A inflação não é um fenômeno meramente monetário, principalmente no Brasil. Não basta controlar a demanda agregada. Também é necessário dar atenção ao lado da oferta. E isso não pode ser feito apenas pela autoridade monetária, que precisa de aliados no combate à inflação. Vale dizer, a estabilidade de preços também depende de outras instâncias responsáveis pela política econômica, além do BCB. 


\section{REGRA DE TAYLOR: BREVE RESENHA}

\section{A literatura internacional}

Taylor (1993) sugere que o processo de condução da política monetária pode ser modelado por uma regra de feedback, que relaciona a taxa básica de juros ao hiato do produto e ao desvio da inflação com relação a uma meta. Ele propõe que o FED determina a taxa de juros de acordo com a seguinte função de reação:

$$
\begin{aligned}
& i_{t}=\alpha_{1}+\alpha_{2}\left(\pi_{t-1}-\pi^{*}\right)+\alpha_{3} y_{t} \\
& \alpha_{1}=\pi_{t-1}+\bar{i}
\end{aligned}
$$

Onde: $i_{t}$ é a taxa básica de juros; $\bar{i}$ é a taxa de juros real de equilíbrio; $\pi_{t-1}$ é a taxa de inflação (acumulada no último ano); $\pi^{*}$ é meta de inflação; e $y_{t}$ é o desvio do PIB real em relação ao potencial;

$$
\text { i.e., } y_{t}=100\left(\frac{P I B_{t}-P I B^{*}}{P I B^{*}}\right) \text {. }
$$

Considerando a taxa de crescimento do PIB potencial dos Estados Unidos igual a $2.2 \%$ e uma meta de inflação de $2 \%$, o autor propõe que a equação (1) deve apresentar os seguintes parâmetros:

$$
i_{t}=\pi_{t-1}+2+0.5\left(\pi_{t-1}-2\right)+0.5 y_{t}
$$

Conforme a equação (2), o FED aumenta a taxa de juros quando: i) a inflação está acima da meta ( $2 \%$ a.a.); e/ou ii) o PIB está crescendo acima de seu potencial $(2.2 \%)$. Repare que uma elevação na inflação gera uma resposta (positiva) mais que proporcional nos juros: um aumento da inflação se reflete em uma ampliação da taxa de juros real. Se ambas as taxas, de inflação e de crescimento do PIB, se igualam às suas respectivas metas, a taxa de juros mantém-se, por construção, em $4 \%$ a.a. (ou $2 \%$ em termos reais). Isto é, $\alpha_{1}$ é a taxa de juros nominal de equilíbrio. ${ }^{1}$

Apesar de a regra de Taylor descrever muito bem o comportamento da taxa de juros dos Estados Unidos, ela não incorpora o que a ortodoxia destaca como um fato estilizado do processo de condução da política monetária: os bancos centrais (BC) tendem a calibrar o juro básico de forma suave e contínua ao longo do tempo. De fato, as autoridades monetárias se mostram avessas a choques de taxas de juros: desvios da inflação e/ou do PIB com relação às suas respectivas metas, usualmente, não implicam resposta drástica e imediata da autoridade monetária,

\footnotetext{
${ }^{1} \mathrm{O}$ termo equilíbrio está sendo usado para denotar a situação em que a autoridade monetária não tem incentivo para alterar a taxa de juros. Isso, por definição, ocorre quando a inflação atinge a meta e o PIB, o seu potencial.
} 
sendo corrigidos gradualmente (Goodfriend, 1987; Mankiw e Miron, 1991; Rudebusch, 1995; Thornton, 2004).

Entre as principais razões para o alto grau de correlação serial da taxa de juros, a teoria ortodoxa destaca: i) o receio de que movimentos abruptos nos juros possam provocar uma crise nos mercados financeiros (Goodfriend, 1991); ii) e a incerteza quanto aos efeitos de variações na taxa de juros (Sack, 1997). No primeiro caso, os BC agiriam de forma gradual para evitar uma eventual crise financeira decorrente de um choque de juros. No segundo, devido ao imperfeito conhecimento do mecanismo de transmissão da política monetária, os BC tomariam suas decisões com base em um processo sequencial de tentativa e erro, implicando a suavização dos movimentos na taxa de juros.

Clarida, Galí e Gertler (1999) incorporam esse fato estilizado da política monetária e propõem importante modificação na regra proposta por Taylor (1993): a introdução de um termo de suavização (ou um componente autorregressivo) que capture a relação entre a taxa de juros e seus valores passados. ${ }^{2}$ Assim, a taxa de juros (corrente) é igual ao seu valor defasado mais um componente à la Taylor, como representado nas equações (3) e (4):

$$
\begin{aligned}
& i_{t}=\alpha_{1} i_{t-1}+\left(1-\alpha_{1}\right) i_{t}^{*} \\
& i_{t}^{*}=\alpha_{2}+\alpha_{3}\left(\pi_{t+1}^{E}-\pi^{*}\right)+\alpha_{4} y_{t+1}^{E} \\
& \alpha_{1} \in(0,1), \alpha_{2}=\pi^{*}+\bar{i}, \alpha_{3}>1, \alpha_{4}>0
\end{aligned}
$$

Substituindo-se (4) em (3):

$$
i_{t}=\alpha_{1} i_{t-1}+\left(1-\alpha_{1}\right)\left[\alpha_{2}+\alpha_{3}\left(\pi_{t+1}^{E}-\pi *\right)+\alpha_{4} y_{t+1}^{E}\right]
$$

O parâmetro $\alpha_{1}$ representa o grau de suavização da taxa de juros. Quanto maior seu valor, maior a inércia na dinâmica (maior a correlação serial da série) da taxa de juros. Observe-se que a equação (5) é uma formulação mais geral do que a originalmente proposta por Taylor: se $\alpha_{1}=0$, a equação (5) se reduz à equação (1). Neste caso, a taxa de juros se ajusta de forma imediata: não há suavização. Contrariamente, se $\alpha_{1} \rightarrow 1$, a taxa de juros se aproxima de um processo autorregressivo de ordem 1 .

Se $\alpha_{3}<1$ ou $\alpha_{4}<0$, a equação (5) é dita desestabilizadora, respectivamente, em relação à inflação e ao PIB (Clarida, Galí e Gertler, 2000). No primeiro caso, o BC acomoda as pressões inflacionárias: uma elevação da inflação implica a redução

\footnotetext{
${ }^{2}$ Para uma abordagem mais formalizada ver Clarida, Galí e Gertler (2000). Eles definem o componente de suavização de forma mais geral como um polinômio nos termos autorregressivos. O modelo estimado na próxima seção se baseia nessa definição.
} 
da taxa de juros real. No segundo, a política monetária é pró-cíclica: um maior crescimento do PIB é acompanhado de uma queda dos juros.

A equação (5) consiste em uma regra do tipo foward-looking, diferentemente da regra de Taylor (1993), que é do tipo backward-looking. De acordo com (5), a taxa de juros se amplia em resposta a um aumento da expectativa de inflação $\left(\pi_{t+1}^{E}\right) \mathrm{com}$ relação à sua meta e a uma elevação do hiato do produto esperado $\left(y_{t+1}^{E}\right)$. Esta equação incorpora de forma mais explícita a concepção de que a existência de defasagens na condução da política monetária (Friedman, 1948) exige um comportamento prospectivo do BC.

De fato, esta formulação é mais geral por permitir que a autoridade monetária forme suas expectativas com base em um conjunto de informação mais amplo, não se baseando apenas nos valores defasados da variável em questão. No entanto, não se trata de uma diferença substancial já que, na ausência de um indicador antecedente confiável para a inflação, suas defasagens podem ser uma boa proxy para a inflação futura. ${ }^{3}$

Clarida, Galí e Gertler (1999) estimam a equação (5) para a economia norte-americana, entre os anos de 1960 e 1996. A Tabela 1 apresenta os parâmetros estimados para duas subamostras, a era pré-Volcker (entre o primeiro trimestre de 1960 e o segundo de 1979) e a era Volcker-Greenspan (do terceiro trimestre de 1979 ao quarto de 1996).

Tabela 1: Função de Reação do FED: 1960/T1 a 1996/T4

\begin{tabular}{c|ccc}
\hline Período & AR $(1)\left(\alpha^{1}\right)$ & Inflação $\left(\alpha^{3}\right)$ & PIB $\left(\alpha^{4}\right)$ \\
\hline \multirow{2}{*}{ Pré-Volcker } & 0.68 & 0.83 & 0.27 \\
& $(0.05)$ & $(0.07)$ & $(0.08)$ \\
Volcker-Greenspan & 0.79 & 2.15 & 0.93 \\
& $(0.04)$ & $(0.40)$ & $(0.42)$ \\
\hline
\end{tabular}

Fonte: Clarida, Galí e Gertler (1999).

Desvio-padrão entre parênteses.

$\mathrm{Na}$ era anterior à gestão de Paul Volcker, a política monetária foi marcadamente acomodatícia. Em média, a taxa de juros real declinava na medida em que a expectativa de inflação se elevava $\left(\alpha_{3}=0.83\right)$. Em contraste com o período anterior, na gestão de Volcker e de Alan Greesnpan, o FED adotou um comportamento claramente proativo. Em média, a taxa de juros real se elevava na medida em que a expectativa de inflação subia $\left(\alpha_{3}=2.15\right)$.

Os artigos aqui resenhados são apenas as principais referências de uma ampla bibliografia. O volume organizado por Taylor (1999) é uma boa coletânea dessa literatura, que é marcadamente ortodoxa. Haight (2008) apresenta uma crítica keynesiana ao que ele acertadamente destaca como sendo a essência da regra de

\footnotetext{
${ }^{3}$ Taylor (1993) obviamente não ignora a existência de defasagens e considera a inflação passada proxy de sua expectativa.
} 
Taylor (1993) - a concepção de que a taxa de juros nominal deve se elevar (reduzir) mais que proporcionalmente em resposta a um aumento (diminuição) da inflação. Para uma visão crítica sobre o tema, ver Rochon (2006). A seguir, a literatura brasileira será tratada.

\section{A literatura brasileira}

Já foram feitas algumas estimativas de regras à la Taylor para a economia brasileira, destacando-se os trabalhos de Figueiredo e Ferreira (2002), Minella et al. (2002), Favero e Giavazzi (2002) e Mendonça (2007). Os principais resultados desses trabalhos serão apresentados neste item.

Antes disso, deve-se registrar que Muinhos (2004) estima regra de Taylor ampliada pela inclusão da taxa de câmbio — proposta por Ball (2000) e Taylor (2001) - , em artigo que visa avaliar o coeficiente de repasse cambial da economia brasileira, após a desvalorização de janeiro de $1999 .{ }^{4}$ Minella et al. (2003) também estimam uma função de reação acrescida da variação cambial. ${ }^{5}$ Cabe ainda mencionar outros três artigos: Andrade e Divino (2001), que usam amostra que vai de 8/1994 a 3/1999; Mendonça (2001), que analisa o período entre o $2^{\circ}$ trimestre de 1996 e o $1^{\circ}$ de 2000; e Salgado, Garcia e Medeiros (2005), que tratam do período entre 1994/7 e 2000/12.

Figueiredo e Ferreira (2002) e Mendonça (2007) dividem a variação do Índice de Preços ao Consumidor Amplo (IPCA) em inflação livre e administrada e mostram que o $\mathrm{BCB}$ reage de forma diferente a estes dois componentes da inflação, que, em suas estimações, são tomados de forma defasada. O BCB reage mais intensamente ao desvio da inflação livre (com relação à meta) do que a uma aceleração da inflação administrada $\left(\alpha_{3}>\alpha_{4}\right)$, como se vê na Tabela 2 .

Tabela 2: Função de Reação do BCB - Figueiredo e Ferreira (2002) e Mendonça (2007)

\begin{tabular}{c|cccccc}
\hline Estudo & Amostra & Constante $\left(\alpha_{1}\right)$ & $\operatorname{AR}(1)\left(\alpha_{2}\right)$ & $\operatorname{Liv}\left(\alpha_{3}\right)$ & $\operatorname{Adm}\left(\alpha_{4}\right)$ & $\mathrm{R}^{2} \mathrm{Aju}$. \\
\hline $\begin{array}{c}\text { Figueiredo } \\
\text { e Ferreira (2002) }\end{array}$ & $1999: 4-2002: 9$ & $\begin{array}{l}0.034^{*} \\
(4.809)\end{array}$ & $\begin{array}{c}0.757^{*} \\
(23.075)\end{array}$ & $\begin{array}{l}0.636^{* *} \\
(2.262)\end{array}$ & $\begin{array}{l}0.498 * * \\
(2.229)\end{array}$ & 0.959 \\
& & & & & & \\
Mendonça & $1999: 1-2004: 11$ & $\begin{array}{l}2.021^{*} \\
(3.131)\end{array}$ & $0.859 *$ & $0.815 *$ & $0.383 * *$ & 0.945 \\
(2007) & & $(20.748)$ & $(2.534)$ & $(3.588)$ & \\
\hline
\end{tabular}

Fonte: elaboração própria com base em Figueiredo e Ferreira (2002) e Mendonça (2007).

Estatística t entre parênteses. * Significativo ao nível de 1\%. * Significativo a 5\%. "Dados em log.

\footnotetext{
${ }^{4}$ Ver também Mishkin (2000) e Holland (2005).

${ }^{5}$ Como a inclusão dessa variável não alterou de forma significativa os resultados de Minella et al. (2002), eles não serão apresentados. Outra variável que também pode ser incorporada é uma proxy para a inclinação da curva de juros, dada pela diferença entre a rentabilidade de dois títulos equivalentes mas com maturidades distintas. Espera-se que haja uma relação inversa entre a inclinação da curva de juros e o nível da Selic.
} 
Minella et al. (2002) e Favero e Giavazzi (2002) empregam regras de Taylor do tipo foward-looking. Favero e Giavazzi (2002) estimam uma função de reação usando a expectativa de variação do IPCA um ano à frente, coletada pelo BCB e publicada no relatório Focus. Sua amostra vai de fevereiro de 1999 a março de 2002. Na primeira linha da Tabela 3 encontram-se os resultados obtidos com apenas 38 observações mensais; a segunda linha refere-se à estimação com base em dados diários.

Tabela 3: Função de Reação do BCB — Favero e Giavazzi (2002): 2/1999 a 3/2002

\begin{tabular}{c|ccc}
\hline Dado & $\operatorname{AR}(1)\left(\alpha_{1}\right)$ & Constante $\left(\alpha_{2}\right)$ & Inflação $\left(\alpha_{3}\right)$ \\
\hline \multirow{2}{*}{ Mensal } & 0.79 & 17.16 & 1.78 \\
& $(0.07)$ & $(0.43)$ & $(0.56)$ \\
\multirow{2}{*}{ Diário } & 0.99 & 17.27 & 1.94 \\
& $(0.003)$ & $(0.41)$ & $(0.58)$ \\
\hline
\end{tabular}

Fonte: elaboração própria com base em Favero e Giavazzi (2002). Desvio-padrão entre parênteses.

Minella et al. (2002) também trabalham com a expectativa de inflação e a meta de inflação doze meses à frente. Eles estimam duas formas funcionais, uma dada pela equação (5) e outra idêntica à equação (5) acrescida de um segundo termo autorregressivo. São usadas duas variáveis dependentes: a taxa Selic e o seu hiato, definido como a diferença entre a Selic e sua tendência, estimada pelo filtro Hodrick-Prescott (HP). Como proxy do hiato do PIB, os autores usam a diferença entre a produção industrial e sua tendência (dada pelo filtro HP).

Cabe destacar que foram utilizadas amostras muito pequenas: a primeira vai de junho de 1999 a junho de 2002 (36 observações); e a segunda compreende o período entre janeiro de 2000 e junho de 2002 (30 observações). Esse número reduzido de observações certamente compromete os resultados obtidos. Na primeira amostra, utilizou-se a expectativa que orienta o Comitê de Política Monetária (Copom) em suas decisões, fornecida pelo BCB em seu Relatório de Inflação. Note que, apesar de significativo, o coeficiente do hiato do produto tem o sinal negativo, diferentemente do esperado (Tabela 4).

Tabela 4: Função de Reação do BCB — Minella et al. (2002): 6/1999 a 6/2002

\begin{tabular}{c|cccccc}
\hline Variável & $\operatorname{AR}(1)\left(\alpha_{1}\right)$ & $\operatorname{AR}(2)\left(\alpha_{2}\right)$ & Constante $\left(\alpha_{3}\right)$ & Inflação $\left(\alpha_{4}\right)$ & $\operatorname{PIB}\left(\alpha_{5}\right)$ & $R^{2}$ Aju. \\
\hline \multirow{2}{*}{ Selic } & $0.76^{*}$ & - & $17.50^{*}$ & $1.78 * *$ & $-0.44 *$ & \multirow{2}{*}{$* .9220$} \\
& $(0.07)$ & - & $(0.36)$ & $(0.84)$ & $(0.11)$ & \\
Selic & $1.04^{*}$ & $-0.20^{*}$ & $17.57^{*}$ & 1.84 & $-0.47 *$ & 0.9342 \\
& $(0.13)$ & $(0.08)$ & $(0.48)$ & $(1.19)$ & $(0.16)$ & \\
Hiato Selic & $0.81^{*}$ & - & $-1.51 *$ & $5.01 *$ & $-0.38 * *$ & 0.9620 \\
& $(0.06)$ & - & $(0.36)$ & $(0.92)$ & $(0.15)$ & \\
Hiato Selic & $1.08^{*}$ & $-0.25^{*}$ & $-1.28^{*}$ & $4.25^{*}$ & $-0.43 *$ & 0.9738 \\
& $(0.09)$ & $(0.06)$ & $(0.36)$ & $(0.77)$ & $(0.13)$ & \\
\hline
\end{tabular}

Fonte: elaboração própria com base em Minella et al. (2002).

Desvio-padrão entre parênteses. * Significativo ao nível de $1 \%$. $^{*}$ Significativo a $5 \%$. 
$\mathrm{Na}$ segunda amostra, foram utilizadas as expectativas de mercado para o IPCA (do relatório Focus). Neste caso, o hiato do produto ou não se mostra significativo ou, quando é significativo, seu coeficiente tem sinal negativo (Tabela 5).

Tabela 5: Função de Reação do BCB — Minella et al. (2002): 1/2000 a 6/2002

\begin{tabular}{c|cccccc}
\hline Variável & $\mathrm{AR}(1)\left(\alpha_{1}\right)$ & $\mathrm{AR}(2)\left(\alpha_{2}\right)$ & Constante $\left(\alpha_{3}\right)$ & Inflação $\left(\alpha_{4}\right)$ & $\mathrm{PIB}\left(\alpha^{5}\right)$ & $\mathrm{R}^{2} \mathrm{Aju}$. \\
\hline \multirow{2}{*}{ Selic } & $0.72^{*}$ & - & $16.49^{*}$ & $1.74^{* *}$ & $-0.25^{* * *}$ & \\
& $(0.08)$ & - & $(0.63)$ & $(0.66)$ & $(0.13)$ & 0.9188 \\
& & & & & & \\
\multirow{5}{*}{ Selic } & $1.36^{*}$ & $-0.56^{*}$ & $16.68^{*}$ & $1.42^{* *}$ & -0.13 & 0.9539 \\
& $(0.18)$ & $(0.15)$ & $(0.69)$ & $(0.72)$ & $(0.17)$ & \\
Hiato Selic & $0.71^{*}$ & - & $-3.28^{*}$ & $3.70 *$ & -0.05 & 0.9694 \\
& $(0.08)$ & - & $(0.54)$ & $(0.58)$ & $(0.13)$ & \\
Hiato Selic & $1.34^{*}$ & $-0.54^{*}$ & $-3.53^{*}$ & $3.63 *$ & 0.08 & 0.9797 \\
& $(0.19)$ & $(0.15)$ & $(0.65)$ & $(0.68)$ & $(0.17)$ & \\
\hline
\end{tabular}

Fonte: elaboração própria com base em Minella et al. (2002).

Desvio-padrão entre parênteses. ${ }^{*}$ Significativo ao nível de $1 \%$. ** Significativo a $10 \%$.

Em resumo, a literatura indica que o $\mathrm{BCB}$, nos primeiros anos de adoção RMI, atuou de forma proativa com relação à inflação. Os artigos resenhados mostram evidência de que uma elevação da inflação gera uma resposta mais que proporcional da Selic: o coeficiente do desvio da inflação com relação à meta varia entre 1.1 (Figueiredo e Ferreira, 2002) e 1.84 (Minella et al., 2002). No entanto, não há evidência de que o $\mathrm{BCB}$ reaja de forma contracíclica ao hiato do produto, como seria esperado. Apenas Minella et al. (2002) usam essa variável em suas regressões, mas o hiato do produto não é significativo ou tem sinal contrário do esperado. ${ }^{6}$

\section{FUNÇÃO DE REAÇÃO DO BCB EM OITO \\ ANOS DE METAS DE INFLAÇÃO (2000-2007)}

\section{Forma funcional e base de dados}

Para avaliar o comportamento do BCB durante a adoção do RMI foi estimada uma regra de Taylor para a economia brasileira do tipo backward-looking. Optou-se por trabalhar com a inflação defasada tendo em vista que a série de expectativas

\footnotetext{
${ }^{6}$ Cabe destacar que Gonçalves e Fenolio (2007) não encontram evidência de manipulação da taxa Selic com fins eleitorais, comprovando que, de facto, o BCB possui independência de instrumentos (Fischer, 1995). Apesar de a relação entre o ciclo político e a política monetária não ser objetivo deste artigo, esse trabalho deve ser referido tendo em vista que a variável hiato do produto é significativa e o seu coeficiente (1.18) tem o sinal esperado (à exceção dos demais). A despeito de se basear em dados relativos ao período de 2000 a 2006, são apenas 28 observações trimestrais, amostra bastante pequena, como as dos demais trabalhos.
} 
de inflação fornecida pelo BCB em seu boletim Focus pode apresentar três graves problemas: i) endogeneidade; ii) causação reversa; e, principalmente, iii) comportamento oportunista - os agentes podem reportar expectativas incorretas com o objetivo de influenciar o comportamento do BCB (Favero e Giavazzi, 2002, p. 10; Gonçalves e Fenolio, 2007, pp. 477-478).

Isso, de forma alguma, implica a negação de que o BCB atua de forma prospectiva, devido às defasagens envolvidas na condução da política monetária. Como será visto adiante, os coeficientes dessa variável são significativos ao nível de $1 \%$ e, portanto, a inflação passada pode ser considerada como uma proxy da inflação futura. Além disso, o $R^{2}$ ajustado da regressão é muito alto, o que significa que a função estimada tem um alto poder explicativo. Neste sentido, pode-se dizer que o BCB usa uma regra de formação de expectativas do tipo adaptativa. ${ }^{7}$

A taxa de variação do IPCA foi dividida em dois componentes - inflação livre e administrada —, com o objetivo de se avaliar se a autoridade monetária continua respondendo de forma distinta a esses dois grupos, como apontado por Figueiredo e Ferreira (2002) e Mendonça (2007). Com vistas a verificar se o BCB é sensível ao lado real da economia, uma proxy do hiato do produto foi incluída na equação (I) estimada:

$$
i_{t}=\alpha_{1} i_{t-1}+\alpha_{2} i_{t-2}+\left(1-\alpha_{1}-\alpha_{2}\right)\left[\alpha_{3}+\alpha_{4}(\overbrace{L v_{t-1}-\pi_{t-1}^{*}}^{\text {Dliv }})+\alpha_{5}(\overbrace{A d m_{t-1}-\pi_{t-1}^{*}}^{\text {Dadm }})+\alpha_{6}(\overbrace{\operatorname{Ind} d_{t-1}-\operatorname{Ind}_{t-1}^{*}}^{\text {Dind }})\right]
$$

onde

$i_{t}$ : taxa Selic efetiva (anualizada) no mês $t$;

$\operatorname{Liv}_{t-1}$ : inflação livre acumulada nos últimos 12 meses até o mês $t$;

$A d m_{t-1}$ : inflação administrada acumulada nos últimos 12 meses até o mês $t$;

$\pi_{t-1}^{*}$ : meta de inflação (últimos 12 meses) no mês $t$;

$I n d_{t-1}:$ taxa de variação (em 12 meses) da produção industrial no mês $t$; e

Ind $d_{t-1}^{*}$ : taxa de variação (em 12 meses) da produção industrial potencial no mês $t .^{8}$

A implantação do RMI, em 21 de junho de 1999, constitui-se em importante mudança de regime, resultando em profunda modificação na condução da política monetária, até então caracterizada pela adoção de um regime monetário de metas

\footnotetext{
${ }^{7}$ Esse fato mostra que, a despeito da importância da revolução das expectativas racionais, como marco da história do pensamento macroeconômico (Modenesi, 2005, cap. 3), do ponto de vista empírico, o conceito de expectativa racional é de restrita aplicação. Assim, ainda que por motivação meramente pragmática, a regra de formação de expectativas adaptativas continua tendo validade empírica.

${ }^{8}$ A taxa Selic é a fornecida pelo Departamento de Mercado Aberto do BCB. Os índices referentes à produção industrial (com ajuste sazonal), aos preços livres e aos administrados foram providos pelo IBGE. As respectivas taxas de variação foram calculadas pelo autor. A taxa de variação da produção industrial mede o crescimento da produção física (quantum) entre o mês $t$ e o mesmo mês do ano anterior. A taxa de variação da produção industrial potencial é obtida pelo filtro HP.
} 
cambiais. Assim, optou-se por excluir os seis primeiros meses de adoção do RMI da amostra, que compreende, então, o período entre janeiro de 2000 e dezembro de 2007.

São 96 observações mensais, número consideravelmente superior ao dos trabalhos acima citados, o que torna as estimativas aqui realizadas mais robustas. Entre eles, Figueiredo e Ferreira (2002) e Mendonça (2007) empregam as maiores amostras, respectivamente, com 42 e 71 observações. Foi incluída uma variável dummy para o mês de setembro de 2003, quando a inflação (acumulada em um ano) representa um outlier.

\section{Testes de raiz unitária}

Para avaliar se as variáveis utilizadas seguem um processo estocástico estacionário, foram realizados dois testes de raiz unitária. A Tabela 6 mostra os resultados do teste Dickey-Fuller Aumentado (ADF). A hipótese nula $\left(H_{0}\right)$, de que a série testada possui raiz unitária (é não estacionária), é rejeitada para as quatro variáveis em questão aos níveis usuais de significância.

Tabela 6: Teste Dickey-Fuller Aumentado

\begin{tabular}{c|ccccc}
\hline Variável & Defasagens & Estatística t & P-Valor & Valor Crítico: $5 \%$ & $10 \%$ \\
\hline Selic & 1 & -2.8215 & 0.0591 & -2.8925 & -2.5834 \\
Dliv & 1 & -2.9125 & 0.0477 & -2.8925 & -2.5834 \\
Dadm & 1 & -1.6404 & 0.0950 & -1.9443 & -1.6145 \\
Dind & 0 & -3.3376 & 0.0159 & -2.8925 & -2.5834 \\
\hline
\end{tabular}

Fonte: elaboração própria.

Seleção de defasagens baseada no critério de informação de Schwarz (SIC).

Adicionalmente, foi realizado o teste de Kwiatkowski, Phillips, Schmidt e Shin (1992) (KPSS), cuja hipótese nula é a de que a série é estacionária. O objetivo de se empregar testes com hipóteses nulas opostas é mitigar o fato de que o teste ADF tem baixo poder (Maddala e Kim, 1998). A Tabela 7 corrobora a evidência de que as séries são estacionárias: para as variáveis Dliv e Dind não se rejeita $H_{0}$ ao nível de significância de $10 \%$; para Selic não se rejeita a $5 \%$; e para Dadm a hipótese de estacionariedade não é rejeitada a $1 \%$.

Tabela 7: Teste KPSS

\begin{tabular}{c|cccc}
\hline Variável & Estatística LM & Valor Crítico: $1 \%$ & $5 \%$ & $10 \%$ \\
\hline Selic & 0.4484 & 0.7390 & 0.4630 & 0.3470 \\
Dliv & 0.2099 & 0.7390 & 0.4630 & 0.3470 \\
Dadm & 0.6821 & 0.7390 & 0.4630 & 0.3470 \\
Dind & 0.0947 & 0.7390 & 0.4630 & 0.3470 \\
\hline
\end{tabular}

Fonte: elaboração própria. 
É provável que a maior dificuldade em se considerar que a variável Dadm seja estacionária decorra do maior grau de inércia dos preços administrados, que seguem a inflação passada mais de perto do que os preços livres. Apesar de exibir um grau de persistência não desprezível, intuitivamente é razoável considerar que Dadm segue um processo estacionário: os choques de preço tendem a se esgotar ao longo do tempo. Este fato, somado à evidência apresentada contrária à hipótese de não estacionariedade, permite considerar que Dadm segue um processo estacionário.

\section{Resultados}

Na Tabela 8 são apresentados os principais resultados da estimação por mínimos quadrados ordinários de três variantes da equação (I). Elas se diferenciam somente quanto às defasagens da variável Dind. Na primeira linha, é usado o seu valor contemporâneo; na segunda, o seu valor defasado; e, na última linha, a segunda defasagem.

Tabela 8: Função de Reação do BCB: 2000-2007

\begin{tabular}{c|ccccccc}
\hline \multirow{2}{*}{ Modelo } & AR (1) $\left(\alpha_{1}\right)$ & AR $(2)\left(\alpha_{2}\right)$ & Constante $\left(\alpha_{3}\right)$ & $\operatorname{Dliv}\left(\alpha_{4}\right)$ & $\operatorname{Dadm}\left(\alpha_{5}\right)$ & $\operatorname{Dind}\left(\alpha_{6}\right)$ & $\mathrm{R}^{2}$ Aju. \\
\hline \multirow{3}{*}{ 1: Dind } & $1.6296^{*}$ & $-0.7115^{*}$ & $14.7140^{*}$ & $0.5158^{*}$ & $0.4171^{*}$ & $0.3200^{* * *}$ & 0.9909 \\
& $(0.0695)$ & $(0.0616)$ & $(0.7088)$ & $(0.1952)$ & $(0.1157)$ & $(0.1712)$ & \\
& & & & & & & \\
2: Dind (-1) & $1.6216^{*}$ & $-0.6987^{*}$ & $14.8107^{*}$ & $0.5370^{*}$ & $0.3862^{*}$ & $0.3301^{* * *}$ & \\
& $(0.0701)$ & $(0.0629)$ & $(0.7474)$ & $(0.2136)$ & $(0.1242)$ & $(0.1933)$ & 0.9909 \\
& & & & & & & \\
3: Dind (-2) & $1.5943^{*}$ & $-0.6700^{*}$ & $14.8284^{*}$ & $0.5765^{*}$ & $0.3677^{*}$ & $0.3957^{* * *}$ & 0.9911 \\
& $(0.0713)$ & $(0.0651)$ & $(0.7510)$ & $(0.2221)$ & $(0.1270)$ & $(0.2133)$ & \\
\hline
\end{tabular}

Fonte: elaboração própria.

Desvio-padrão entre parênteses. ${ }^{*}$ Significativo ao nível de $1 \%$. ** Significativo a $10 \%$.

Entre as três variantes desse modelo, a (2) apresenta melhores propriedades estatísticas. Em particular, o teste Breusch-Godfrey de correlação serial dos resíduos (LM) é mais favorável ao modelo (2) do que ao modelo (1). Para o modelo (3), é mais difícil não rejeitar a hipótese nula de inexistência de correlação serial.

Para efeito de análise, o modelo (2) foi selecionado e doravante será referido, simplesmente, como o modelo. No Gráfico 1, em que se encontram a Selic estimada e a observada, vê-se que a função de reação estimada representa bem o comportamento do BCB. Esta alta aderência decorre de um elevado $R^{2}$ ajustado (0.99), como também se verifica nos artigos resenhados (entre 0.92 e 0.98 ). 
Gráfico 1: Taxa Selic Observada e Estimada

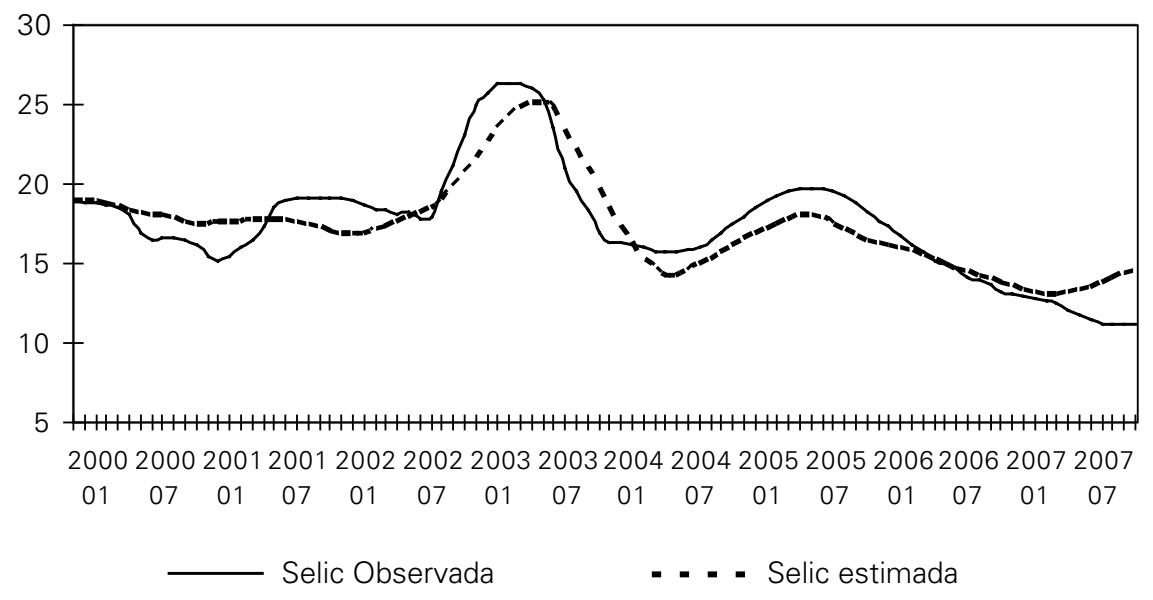

Fonte: elaboração própria.

Como foi usada amostra consideravelmente diferente dos trabalhos resenhados foram obtidos resultados bastante distintos. A principal diferença diz respeito ao grau de inércia da taxa juros, que se mostrou extremamente elevada. A inércia da taxa de juros é medida pelo peso do(s) componente(s) autorregressivo(s). No modelo estimado, a soma dos coeficientes dos dois termos autorregressivos aproxima-se de $1\left(\alpha_{1}+\alpha_{2}=0.92\right)$, implicando elevado grau de autocorrelação na taxa de juros. Nos trabalhos resenhados, o peso do(s) componente(s) autorregressivo(s) é um pouco menor, variando entre 0.72 e 0.86 .

Ao determinar a Selic, o Copom dá pouca relevância ao estado da economia, ou seja, à situação inflacionária (Dliv e Dadm) e ao grau de aquecimento da economia (Dind). Um elevado grau de suavização resulta em reduzida sensibilidade ao estado da economia: diante de uma queda (elevação) da inflação e/ou de uma desaceleração (aceleração) da economia, o BCB reage de forma muito gradual, reduzindo (aumentando) os juros de forma demasiadamente lenta e parcimoniosa.

Outra diferença relevante refere-se ao hiato do produto, que é significativo (ao nível de $10 \%)$ e o seu coeficiente $\left(\alpha_{6}=0.33\right)$ tem o sinal esperado. Em Minella et al. (2002), único trabalho em que esta variável também foi incluída, seu coeficiente não é significativo ou tem sinal negativo. Entretanto, a baixa magnitude do coeficiente do hiato do produto indica que o BCB é pouco sensível ao lado real da economia. Cabe notar que, durante a era Volcker-Greenspan, o FED mostrou-se muito mais preocupado com o crescimento econômico $\left(\alpha_{4}=0.93\right)$ do que as autoridades monetárias brasileiras, no período analisado.

$\mathrm{O} \mathrm{BCB}$ parece continuar reagindo mais intensamente em relação à inflação livre $\left(\alpha_{4}=0.54\right)$ do que à administrada $\left(\alpha_{5}=0.39\right)$. A diferença entre os coeficientes $\left(\alpha_{4}\right.$ $\left.-\alpha_{5}=0.15\right)$ é praticamente igual à encontrada por Figueiredo e Ferreira (2002) e consideravelmente menor do que a apresentada por Mendonça (2007), de 0.43.

Como a autoridade monetária não influencia diretamente os preços adminis- 
trados, o controle da inflação se concentrou na repressão dos preços livres. Assim, a relação entre os preços administrados e os livres aumentou $30 \%$, entre os anos de 2000 e 2007 (Gráfico 2). Não cabe aprofundar aqui essa questão, contudo deve-se lembrar que essa expressiva mudança de preços relativos tende a gerar distorções alocativas não desprezíveis.

Gráfico 2: Índice de Preços Relativos (Administrados/Livres): 1/2000 a 12/2007

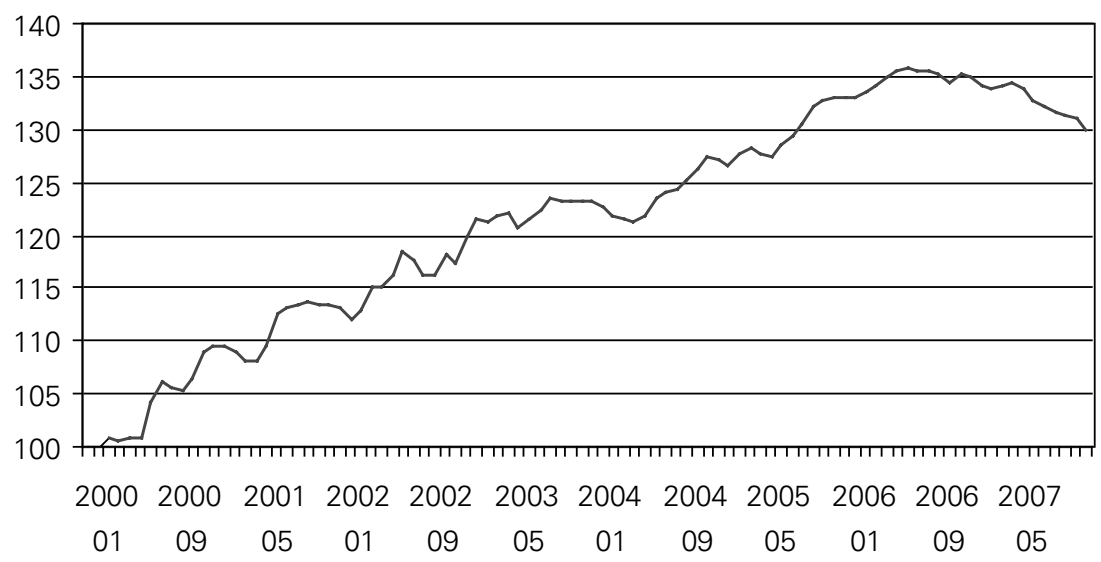

Fonte: elaboração própria. Base: $2000=100$.

Finalmente, como em todos os artigos resenhados, a constante é positiva e de grande magnitude ( $\alpha_{3}=14.81$ ), indicando uma elevada taxa de juros (nominal) de equilíbrio. Como será visto na próxima seção, esta característica da função de reação do BCB é peça fundamental do conservadorismo que marca a política monetária no Brasil.

\section{O CONSERVADORISMO NA POLÍTICA MONETÁRIA}

A evidência aqui apresentada corrobora a ideia de que a formação da taxa Selic é marcada por um excesso de conservadorismo. ${ }^{9}$ Tal proposição se sustenta

\footnotetext{
${ }^{9}$ Bresser e Nakano (2002) e Nakano (2006) se inspiram no conceito de convenção (Keynes, 1936, 1937) para encontrar justificativa para a manutenção da taxa Selic em níveis excessivamente elevados. Segundo Nakano (2006), a inflação estaria sob controle e também não haveria pressão de demanda. Assim, a lentidão no processo de redução da Selic (iniciado em 2005) somente se justificaria pela aceitação por parte do BCB de convenção estabelecida pelos agentes econômicos de que: i) existe um piso consideravelmente elevado para os juros; e ii) a política monetária deve-se basear em uma regra de Taylor. Erber (2008a, 2011) vai além, ao considerar que o excesso de rigidez monetária somente pode ser entendido sob o prisma da economia política. Não se trata de uma questão puramente macroeconômica, mas, sim, o resultado de uma coalizão de interesses formada em torno da manutenção dos juros em níveis elevados. Sobre o conceito de convenção, ver Chavance (2007) e
} 
com a constatação de que a função de reação do $\mathrm{BCB}$ possui duas características marcantes: i) o alto peso dos componentes auto-regressivos; e, notadamente, ii) uma taxa de juros de equilíbrio consideravelmente alta.

O BCB tem levado longe demais a crença ortodoxa - que, embora questionável, não se propõe aqui discutir - de que os movimentos nos juros devem ser graduais. O peso dos componentes autorregressivos, na função de reação do BCB $\left(\alpha_{1}+\alpha_{2}=0.92\right)$, é bastante superior ao peso do componente autorregressivo na função de reação do FED, tanto na era pré-Volcker $\left(\alpha_{1}=0.68\right)$, quanto no período Volcker-Greenspan $\left(\alpha_{1}=0.79\right)$.

A contrapartida do alto peso do componente autorregressivo é uma reduzida importância dada pelo BCB ao estado da economia. No caso brasileiro, as autoridades monetárias são muito pouco sensíveis ao comportamento da inflação e ao nível de atividade $\left(1-\alpha_{1}-\alpha_{2}=0.08\right)$; diferentemente do FED, que dá mais atenção à situação econômica $\left(1-\alpha_{1}=0.21\right)$ ao tomar suas decisões.

Afirmar que o $\mathrm{BCB}$ conduz a política monetária com base na crença ortodoxa de que os movimentos na taxa de juros devem ser suaves não é exatamente uma novidade. Essa postura é defendida publicamente pela instituição e já se tornou de domínio público o entendimento de que o $\mathrm{BCB}$ é conservador. O que surpreende é o grau de suavização praticado pelo BCB. Os resultados mostram um BC extremamente avesso a realizar movimentos - ainda que pequenos - nos juros. Assim, mesmo diante de uma melhora do cenário inflacionário e/ou uma desaceleração econômica, a probabilidade de a taxa Selic ficar inalterada é bastante alta.

A elevada inércia da Selic também pode ser constatada na Tabela 9, que apresenta as variações absolutas da taxa básica por faixa de magnitude. O Copom se mostrou relutante em alterar a Selic em mais do que 0.5 p.p., em cada oportunidade. Entre janeiro de 2000 e dezembro de 2007, o Comitê se reuniu 91 vezes. Em $40 \%$ delas, a Selic ficou inalterada. As variações de até 0.5 p.p. representam $82 \%$ do total. A taxa variou mais de 1.0 p.p. em menos de $8 \%$ das vezes em que o Comitê se reuniu. Não houve mudança superior a 3.0 p.p., que ocorreram em apenas $2 \%$ das reuniões.

Tabela 9: Variações da Selic por Faixa de Magnitude: 2000-2007

\begin{tabular}{c|ccc}
\hline $\begin{array}{c}\text { Magnitude } \\
\text { Absoluta (p.p.) }\end{array}$ & $\begin{array}{c}\text { Frequência } \\
\text { Absoluta }\end{array}$ & $\begin{array}{c}\text { Frequência } \\
\text { Relativa }\end{array}$ & $\begin{array}{c}\text { Frequência Relativa } \\
\text { Acumulada }\end{array}$ \\
\hline 0.00 & 36 & 39.6 & 39.6 \\
0.25 & 12 & 13.2 & 52.8 \\
0.50 & 26 & 28.6 & 81.4 \\
0.75 & 5 & 5.5 & 86.9
\end{tabular}

Dequech (2008). Erber (2008b) trata do caso brasileiro. Para uma resenha sobre o tema, ver Modenesi e Modenesi (2010). 


\begin{tabular}{c|ccc}
1.00 & 5 & 5.5 & 92.4 \\
1.50 & 3 & 3.3 & 95.7 \\
2.00 & 1 & 1.1 & 96.8 \\
2.50 & 1 & 1.1 & 97.9 \\
3.00 & 2 & 2.2 & 100.0 \\
Total & 91 & 100 & - \\
\hline
\end{tabular}

Fonte: elaboração própria com dados do BCB.

Contudo, a inércia não é suficiente para explicar o conservadorismo na política monetária. Com efeito, a inércia é simétrica: uma elevação da inflação — ou do hiato do produto - também não ocasiona alta expressiva e abrupta da taxa básica.

O BCB não é apenas avesso a alterar a Selic. O principal elemento do seu conservadorismo é o elevado nível da taxa de juros (nominal) de equilíbrio estimada. Se a meta de inflação for cumprida $(D l i v, D a d m=0)$ e o produto se igualar ao seu potencial $(\operatorname{Dind}=0$ ) sistematicamente, a Selic converge para um valor bastante alto, cerca de $15 \%$ (ceteris paribus)..$^{10}$ Nesta hipótese, em dez meses, a Selic atinge valor perto de $15 \%$; em 35 meses, ela alcança $14.81 \%$, estabilizando-se neste nível (Gráfico 3). Ou seja, com o cumprimento estrito e contínuo da meta de inflação e um perfeito equilíbrio entre oferta e demanda agregada, por oito anos seguidos, o BCB manteria a Selic em torno de $15 \%$ a.a.

Gráfico 3: Selic Observada e Convergência ao Equilíbrio (Dliv, Dadm, Dind =0)

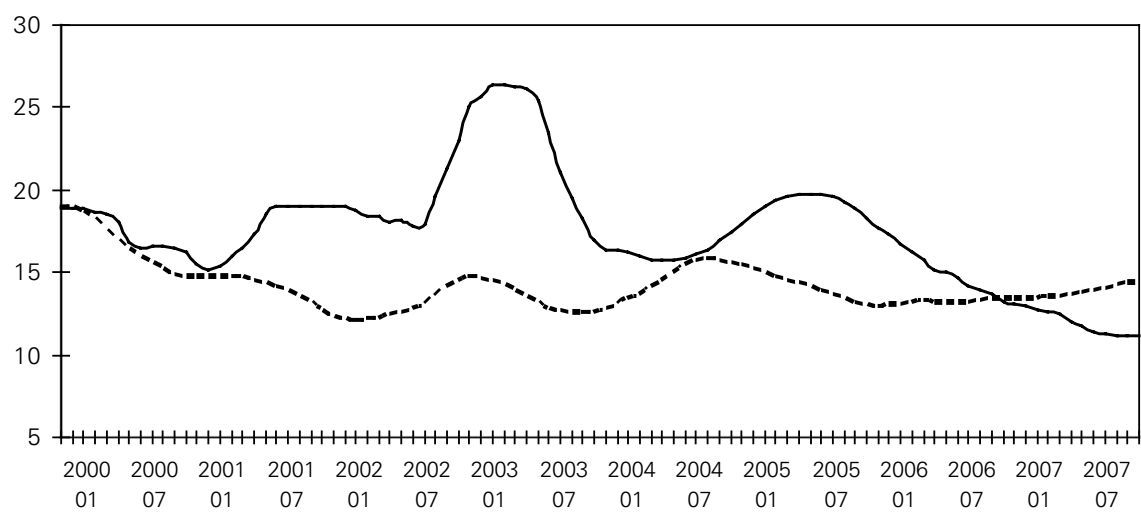

_ Selic Observada _. - Selic estimada (Dliv, Dadm = -1)

Fonte: elaboração própria.

\footnotetext{
${ }^{10}$ As simulações apresentadas supõem a constância de todos os parâmetros relevantes, notadamente os da função de reação do BCB. Não constituem modelo de previsão da Selic.
} 
O elevado patamar da taxa de juros de equilíbrio conjugado com o excessivo grau de suavização praticado pelo BCB faz com que a Selic flutue próximo - e dificilmente se desvie - de um valor muito alto. O conservadorismo do BCB também pode ser visualizado no Gráfico 4. Nele, encontram-se a taxa Selic observada e a Selic estimada que vigoraria caso, ambos, os desvios da inflação livre e da administrada com relação à meta, fossem iguais a menos 1 p.p. (Dliv, Dadm $=-1)$, em todos os 96 meses do período analisado (ceteris paribus).

Gráfico 4: Simulação 1: Taxa Selic Observada e Estimada (Dliv, Dadm = - 1)

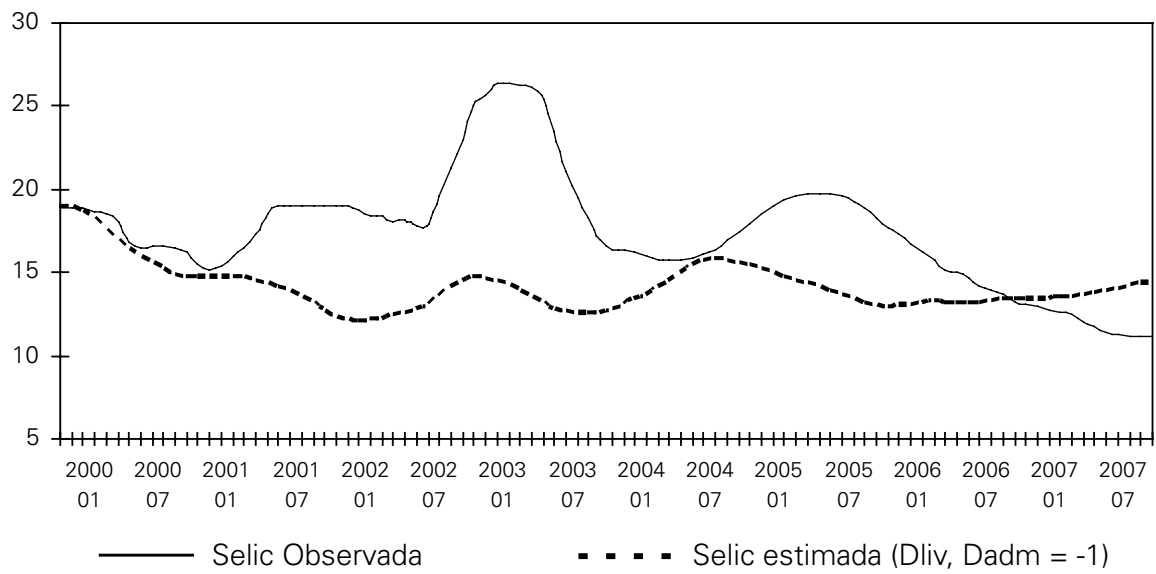

Fonte: elaboração própria.

Mesmo em um cenário em que a inflação se mantém absolutamente controlada - com o IPCA permanentemente abaixo do centro da meta por oito anos seguidos - , a taxa Selic não teria sido significativamente menor do que a observada, como naturalmente se esperaria. Pelo contrário, em média, a Selic estimada teria sido de $14.18 \%$, somente 3.34 p.p. abaixo da Selic observada (17.52\%).

Para que houvesse uma queda expressiva da taxa básica, por exemplo, para cerca de $6 \%$, a inflação deveria ser 10 p.p. menor que o centro da meta (Dliv, Da$d m=-10$ ). Neste caso, em doze meses a Selic converge para valor próximo a $6 \%$; a partir de então, ela oscila em torno deste patamar (ceteris paribus). ${ }^{11}$ Isto é, seria necessária uma deflação crônica e de grandes proporções para que o BCB fixasse a Selic em um patamar razoável, aproximando-a das taxas praticadas no resto mundo (Gráfico 5).

\footnotetext{
${ }^{11}$ Trata-se de algo absolutamente inverossímil. Por exemplo, para que essa hipótese se verificasse em dezembro de 2007 , quando a meta de inflação era de $4.5 \%$, o IPCA deveria ter apresentado deflação de $5.5 \%$.
} 
Gráfico 5: Simulação 2: Taxa Selic Observada e Estimada (Dliv, Dadm = - 10)

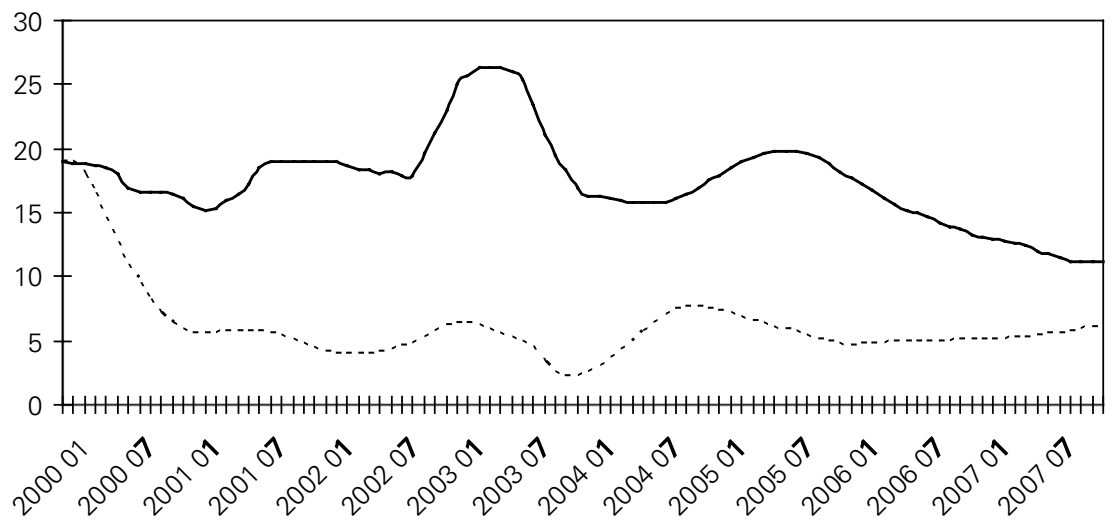

Selic Observada

- - - Selic estimada (Dliv, Dadm $=-10.0 \%$ )

Fonte: elaboração própria.

Em síntese, o conservadorismo na condução da política monetária se materializa em duas características distintivas da função de reação do BCB: o alto peso dos componentes autorregressivos; e, sobretudo, uma taxa de juros de equilíbrio muito elevada. Isso significa que, mantido o quadro atual, a taxa de juros dificilmente se reduziria de forma satisfatória.

\section{CONSIDERAÇÕES FINAIS}

O processo de estabilização monetária, iniciado em 1994 com o Plano Real, somente estará concluído de forma definitiva com a reversão do quadro aqui traçado. A primeira fase desse processo - a eliminação da alta inflação crônica e a consequente estabilização dos preços - foi alcançada com sucesso há uma década e meia. Entretanto, o alto custo que a sociedade brasileira vem pagando pela estabilidade de preços impede que essa conquista - uma das mais relevantes de nossa história econômica contemporânea - se torne perene ou politicamente sustentável.

Falta, ainda, a última etapa do processo de estabilização: a flexibilização da política monetária. Já não é mais suficiente controlar a inflação praticando as maiores taxas de juros do mundo. É neste sentido que, no caso brasileiro, o RMI tem-se mostrado pouco adequado. A política anti-inflacionária tem resultado em elevadíssima taxa de sacrifício - medida não apenas pelo alto desemprego e o relativo desaquecimento da economia, mas, também, pelo enorme custo fiscal da política monetária. A estabilidade somente estará consolidada - tanto do ponto de vista econômico quanto político - quando o controle da inflação estiver combinado com uma política monetária menos rígida e, portanto, sustentável a longo prazo. 
Alguns consideram que a redução da Selic é meramente uma questão de vontade. Outros rechaçam o que identificam como um voluntarismo ingênuo e ressaltam que é necessária a criação de precondições objetivas para que a flexibilização da política monetária não implique retomada da inflação. Esse é um debate que ainda precisa ser aprofundado. É aí que se encontrará a solução definitiva para a interrupção do processo de stop and go - instaurado há quase três décadas - e a consequente retomada sustentável do crescimento econômico do país.

$\mathrm{O}$ avanço desse debate deve ocorrer em duas frentes. A primeira é a mensuração da taxa de sacrifício da política anti-inflacionária. É necessário avaliar o impacto de uma variação na Selic sobre os preços vis-à-vis seus efeitos perversos sobre os níveis de investimento, PIB, emprego, endividamento público e a taxa de câmbio.

A segunda vertente do debate consiste em uma investigação mais detalhada do mecanismo de transmissão da política monetária, com o intuito de se identificar suas peculiaridades. De fato, o conservadorismo do BCB tem sido justificado em função de problemas no mecanismo de transmissão: o entupimento de determinados canais demandaria uma dose excessivamente elevada de juros para manter a estabilidade de preços.

Mas a existência de problemas na transmissão da política monetária não deve ser justificativa para a fixação da Selic em níveis demasiadamente altos, em face da elevada taxa de sacrifício que a estratégia vigente tem cobrado da economia e da sociedade brasileira. Mesmo se comprovada a reduzida eficácia da política monetária, não se justifica continuar insistindo em uma terapia cujos custos são muito elevados relativamente aos seus benefícios.

A possibilidade de mudar a estratégia de estabilização de preços deve ser seriamente considerada. Uma vez que os juros se revelam pouco potentes para controlar os preços, faz-se necessária a utilização de outros instrumentos no combate à inflação. Se há problemas na transmissão da política monetária, não cabe apenas ao $\mathrm{BCB}$ a tarefa de controlar os preços. Ou seja, não seria adequado delegar, exclusivamente, ao BCB a função de guardião da estabilidade. O BCB precisa de aliados no cumprimento de uma função que ele, sozinho, não se tem mostrado apto a cumprir de forma satisfatória.

\section{REFERÊNCIAS BIBLIOGRÁFICAS}

ANDRADE, J. P., DIVINO, J. A. C.A. (2001), “Optimal rules for monetary policy in Brasil”. Instituto de Pesquisa Econômica Aplicada, Texto para Discussão, 806.

BALL, L. (2000), "Policy rules and external shocks". NBER Working Paper Series, 7910, September.

BRESSER-PEREIRA, L. C. e NAKANO, Y. (2002), "Uma Estratégia de Desenvolvimento com Estabilidade”. Revista de Economia Política, vol. 22 (3), pp. 146-180.

CHAVANCE, B. (2007), L'économie Institutionnelle. Paris: La Découverte.

CLARIDA, R., GALÍ, J. e GERTLER, M. (1999), “The science of monetary policy: a new Keynesian perspective". Journal of Economic Literature, vol. 37, pp. 1661-1707.

CLARIDA, R., GALÍ, J. e GERTLER, M. (2000), "Monetary policy rules and macroeconomic stability: Evidence and some theory”. The Quarterly Journal of Economics, vol. 115, pp. 147-180. 
DEQUECH, D. (2008), “Conventions, conformity, and deviation: a preliminary discussion”. Kansas City, Anais da 10th Internacional Post Keynesian Conference.

FAVERO, C.A., e GIAVAZZI, F. (2002), “Why are Brazil's interests rates so high?” Università Bocconi, Innocenzo Gasparini Institute for Economic Research, Working Paper, 224.

ERBER, F. (2008a), "Development projects and growth under finance domination - the case of Brazil during the Lula years (2003-2007)". Review Tiers Monde, 194 (no prelo).

ERBER, F. (2008b), “The Evolution of Development Conventions”. Instituto de Economia, Universidade Federal do Rio de Janeiro. XII ISS Conference, Rio de Janeiro, Julho.

ERBER, F. (2011), “As convenções de desenvolvimento no Governo Lula”. Revista de Economia Política, vol. 31(1), pp. 31-55.

FIGUEIREDO, F.M.R. e FERREIRA, T.P. (2002), "Os preços administrados e a inflação no Brasil”. Banco Central do Brasil, Trabalhos para Discussão, 59.

FISCHER, S. (1995), “Central bank independence revisited”. American Economic Review, vol. 85 (2), May, pp. 201-206.

FRIEDMAN, M. (1948), “A Monetary and Fiscal Framework for Economic Stability”. The American Economic review, vol. XXXVIII (3), pp. 245-264.

GONÇALVES, C.E.S, e FENOLIO, F.R. (2007), “Ciclos eleitorais e política monetária: evidências para o Brasil”. Instituto de Pesquisa Econômica e Aplicada, Pesquisa e Planejamento Econômico, vol. 37 (3), pp. 465-487.

GOODFRIEND, M. (1987), "Interest rate smoothing and price level trend-stationary". Journal of Monetary Economics, 19, pp. 335-348.

GOODFRIEND, M. (1991), "Interest rate and the conduct of monetary policy". In: Anais do Carnegie-Rochester Conference Series on Public Policy, 34, pp. 7-30.

HAIGHT, A.D. (2008), “A Keynesian angle for the Taylor rule: mortgage rates, monthly payment illusion, and the scarecrow effect of inflation". Journal of Post Keynesian Economics, vol. 30 (2), winter, pp. 259-277.

HOLLAND, M. (2005), "Monetary and exchange rate policy in Brazil after inflation targeting”. XXXIII Encontro Nacional de Economia, ANPEC.

KEYNES, J.M. (1936), The General Theory of Employment, Interest And Money. London: Macmillan.

KEYNES, J.M. (1937), “The general theory of employment”. The quarterly Journal of Economics, vol. 51 (2), pp. 209-223.

KWIATKOWSKI, D., PHILLIPS, P.C.B., SCHMIDT, P. e SHIN, Y. (1992), “Testing the Null Hypothesis of Stationary against the Alternative of a Unit Root". Journal of Econometrics, 54, pp. 159178.

MADDALA, G.S., KIM, I.M. (1998), Unit Roots, Cointegration and Structural Change. Cambridge (UK): Cambridge University Press.

MANKIW N. G. e MIRON J. A. (1991), "Should the FED Smooth Interest Rates? The case of Seasonal Monetary Policy”. Carnegie- Rochester Conference Series on Public Policy, 34, pp. 41-70.

MINELLA, A., FREITAS, P.S., GOLDFAJN, I. e MUINHOS, M.K. (2002), "Inflation targeting in Brazil: Lessons and challenges”. Banco Central do Brasil, Trabalhos para Discussão, 53.

MINELLA, A., FREITAS, P.S., GOLDFAJN, I. e MUINHOS, M.K. (2003), "Inflation Targeting in Brazil: Constructing Credibility under Exchange Rate Volatility". Journal of International Money and Finance, vol. 27 (7), pp. 1015-104.

MENDONÇA, H.F. (2001), "Mecanismos de transmissão monetária e a determinação da taxa de juros: uma aplicação da regra de Taylor ao caso brasileiro". Economia e Sociedade, 16, pp. 65-81.

MENDONÇA, H.F. (2007), "Metas de inflação e taxa de juros no Brasil: uma análise do efeito dos preços livres e administrados”. Revista de Economia Política, 27, pp. 431-451.

MISHKIN, F. (2000), "Inflation targeting in emerging markets countries". NBER Working Paper Series, 7618.

MODENESI, A.M. (2005), Regimes Monetários: Teoria e a Experiência do Real. Barueri: Manole.

MODENESI, A.M. e MODENESI, R.L. (2010). "Quinze Anos de Rigidez Monetária no Brasil (1995- 
-2008): uma agenda de pesquisa”. III Encontro Internacional da Associação Keynesiana Brasilei$\mathrm{ra}$, UFRGS.

MUINHOS, M.K. (2004), "Inflation targeting in an open financially integrated emerging economy: The case of Brazil”. Estudos Econômicos, vol. 34 (2), 269-296.

NAKANO, Y. (2006), “Antes que os populistas tomem conta”. Jornal Valor Econômico, 17/1/2006.

ROCHON, L.-P. (2006). "The more things change... inflation targeting and central bank policy". Journal of Post Keynesian Economics, Summer, vol. 28 (4), pp. 551-558.

RUDEBUSCH, G. D. (1995), "Federal Reserve Interest Rate Targeting, Rational Expectations and Term Structure”. Journal of Monetary Economics, 35, pp. 245-274.

SAlGADO, M. J. S, GARCIA, M. G. P, e MEDEIROS, M. C. (2005), "Monetary Policy During Brazil's Real Plan: Estimating the Central Bank's Reaction Function”. Revista de Economia Política, pp. 61-79.

SACK, B. (1997), "Uncertainty and gradual monetary policy", Board of Governors of the Federal Reserve System. Mimeografado.

STIGLITZ, J. (2008), “A falência das metas de inflação”. O Globo, Opinião, p. 10, 7 de junho.

TAYLOR, J.B. (1993), "Discretion versus policy rules in practice”, Carnegie-Rochester Conference Series on Public Policy, 39, pp. 195-214.

TAYLOR, J.B. (Ed.) (1999), Monetary Policy Rules. Chicago: Chicago University Press.

TAYLOR, J.B. (2001), "The role of exchange rates in monetary-policy rules". American Economic Review, vol. 90 (1), pp. 263-267.

THORNTON, D. L. (2004), "The FED and Short-Term Rates: Is it Open Market Operations, Open Mouth Operations or Interest Rate Smoothing?”. Journal of Banking \& Finance 28, pp. 475-498. 